\title{
Motions of one stiff molecule in an entangled polymer melt
}

\author{
P. G. de Gennes \\ Collège de France, 75231 Paris Cedex 05, France \\ (Reçu le 3 octobre 1980, accepté le 24 novembre 1980)
}

\begin{abstract}
Résumé. - 1) Une molécule en bâtonnet, de longueur $L$, immergée dans un polymère fondu formé de chaînes flexibles ( $P$ monomères par chaîne) doit avoir une mobilité de translation proportionnelle à $L^{-1}$ et indépendante de $P$. 2) Le coefficient de diffusion de rotation $D_{\text {rot }}$ est plus délicat :a) pour $L$ grand $D_{\text {rot }} \sim k T /\left[\eta(P) L^{3}\right]$ doit être contrôlé par la viscosité macroscopique $\eta(P)$ du fondu; $b$ ) pour $L$ moins grand $D_{\text {rot }}$ deviendrait proportionnel à $L^{-5}$ et indépendant de $P$ : dans ce régime, la molécule rigide glisse le long de son axe et réalise de faibles changements d'orientation à chaque glissement (« reptation-rotation »). Nous discutons les conséquences de ces propriétés sur les régimes transitoires d'électrophorèse, sur l'effet Kerr et sur la viscoélasticité linéaire de suspensions diluées.

Abstract. - 1) A rod, of length $L$ immersed in a sea of flexible polymer chains ( $P$ monomers/chain) is expected to have a translational mobility (or diffusion constant) inversely proportional to the rod length and independent of $P$. 2) The rotational diffusion constant $D_{\text {rot }}$ is more delicate $: a$ ) for very long rods $D_{\text {rot }} \sim k T /\left[\eta(P) L^{3}\right]$ is controlled by Stokes friction and inversely proportional to the melt viscosity $\eta(P) ; b)$ for shorter rods, $D_{\text {rot }}$ becomes proportional to $L^{-5}$ and independent of $P$. Regime $(b)$ describes a " reptation-rotation process " where the rod slides along its own axis and achieves weak, cumulative, changes in orientation. We discuss the consequences of these properties on the Kerr effect, and on the linear viscoelastic properties, of dilute suspensions of rods.
\end{abstract}

1. Aim and model. - Experimental studies on rod molecules immersed in an entangled polymer solution (or melt) are not very numerous [1]. The main difficulty, even for very dilute rods, is to avoid segregation of the solutes [2]. Another, possibly more favourable situation from this point of view, is obtained with stiff molecules moving inside a network - gel electrophoresis of DNA fragments being a typical example. It is of some interest to understand, at least qualitatively, the stochastic motions of one such rod in its entangled environment. A similar study has been carried out recently for long flexible solutes in a melt [3]. We shall see here that the case of stiff solutes is very different. We use the simplest model. A structureless rod, of length $L=N a$ ( $N$ being the number of monomer units) is immersed in a dense melt of flexible chains : each chain has the same degree of polymerization $P$, and an unperturbed end to end distance $R_{0}(P)=P^{1 / 2} a$. We also write the monomer volume as $a^{3}$ : this arbitrary assumption being made to reduce the number of independent lengths in the problem. We proceed similarly for the time scales : the friction processes involving flexible monomers, or rod monomers, are described by a single constant, for instance a microscopic viscosity $\eta_{0}$, or a related jump time $\tau_{0}=\eta_{0} a^{3} / k T$ ( $T$ being the temperature).

The only numerical constant which we want to retain is the average number $N_{\mathrm{e}}$ of monomers between consecutive entanglement points on a $P$ chain. The importance of this number $N_{\mathrm{e}} \sim 200$ is stressed in various reviews [4]. For instance, if the pure melt is driven elastically at not too low frequencies $\omega$, it behaves like a rubber, with elastic moduli of order

$$
E_{0}=\frac{k T}{N_{\mathrm{e}} a^{3}} .
$$

This holds when $\omega \tau(P)>1$, where $\tau(P)$ is the terminal relaxation time of the melt. In a simple reptation picture [5] we have

$$
\tau(P) \cong \tau_{0} P^{3} N_{\mathrm{e}}^{-1} \quad\left(P>N_{\mathrm{e}}\right) .
$$

The bulk viscosity of the melt is then

$$
\eta(P) \cong E_{0} \tau(P) \cong \eta_{0} P^{3} N_{\mathrm{e}}^{-2}
$$

We constantly assume that the rod diameter is much smaller than the spatial distance $R_{\mathrm{e}}$ between entanglement points $R_{\mathrm{e}}=N_{\mathrm{e}}^{1 / 2} a$.

In our model, we assume the rod diameter to be $\sim a$, and the assumption is equivalent to $N_{\mathrm{e}} \gg 1$. 
2. Translational mobility. - One approach for the evaluation of the rod mobility would be based on continuum fluid mechanics : if a needle of length $L$ is immersed in a fluid of bulk viscosity $\eta(P)$, it would show a mobility along its axis [6]

$$
\mu_{\|}=[2 \pi L \eta(P) \ln (L / a)]^{-1}
$$

and a mobility transverse to the axis $\mu_{\perp}=1 / 2 \mu_{\|}$. However, for our purposes, equation (2.1) is wrong. The rod, when sliding along its axis, experiences not the global viscosity $\eta(P)$ but rather a local viscosity of order $\eta_{0}$. Thus we shall write instead of (2.1) (omitting all numerical factors, logs, etc.)

$$
\mu_{\|} \cong \frac{1}{L \eta_{0}} .
$$

This type of law was first derived some time ago by H. Frisch $\left({ }^{1}\right)$. The large difference between the Stokes value (2.1) and the Frisch value (2.2) is conceptually related to a recent discussion of boundary conditions for the flow of polymer melts near a flat, passive wall [7]. As shown in reference [7] the boundary conditions allow for a significant slip at the wall, when the ambient fluid is strongly entangled.

We should also emphasize that equation (2.2) assumes that there are no bonds established between the rod and the molten chains : this assumption is contained in our statements of section 1 , where a single friction coefficient is incorporated in the model.

It is also essential to realize that the transverse mobility $\mu_{\perp}$ is enormously smaller than $\mu_{\|}$. Because any transverse motion on distances larger than $R_{\mathrm{e}}$ requires some disentanglement among the neighbouring chains, we are led to predict $\mu_{\perp} \sim(L \eta(P))^{-1}$. This mobility is so small that it is probably inaccessible to experiments.

If the angle $\theta$ (between the rod and the direction of the driving force) is distributed at random, we expect an overall mobility

$$
\begin{aligned}
& \mu_{\text {trans }}=\left\langle\cos ^{2} \theta\right\rangle \mu_{\|}+\left\langle\sin ^{2} \theta\right\rangle \mu_{\perp} \cong \\
& \cong\left\langle\cos ^{2} \theta\right\rangle \mu_{\|}=\frac{1}{3} \mu_{\|} .
\end{aligned}
$$

But, of course, for many practical applications, the distribution of $\theta$ may be anisotropic : for instance if the driving force is an electric field aligning the rods. Other effects related to drift with a distribution of orientations will be discussed in section 4 .

3. Reorientation processes. - 3.1 REPTATION-RoTATION. - At any instant the rod is trapped in a tube of length $L$, and diameter $\sim R_{\mathrm{e}}=N_{\mathrm{e}}^{1 / 2} a$. The angular fluctuations which are thus allowed are of order :

$$
\psi=\frac{R_{\mathrm{e}}}{L} .
$$

( $\left.{ }^{1}\right)$ Unpublished work.
After a certain time $T_{\text {rep }}$ the rod has completely moved off the original tube and has thus chosen a slightly different orientation : the amount of angular freedom is $\psi$, and the resulting rotational diffusion constant is :

$$
D_{\text {rot }} \cong \frac{\left\langle\psi^{2}\right\rangle}{T_{\text {rep }}}=\frac{N_{\mathrm{e}} a^{2}}{T_{\text {rep }} L^{2}} .
$$

The evaluation of $T_{\text {rep }}$ follows a classical argument : inside its own tube the rod moves back and forth, and this Brownian motion is described by a tube diffusion constant :

$$
D_{\mathrm{t}}=k T \mu_{\|} \cong \frac{k T}{\eta_{0} L} .
$$

The time $T_{\text {rep }}$ required for leaving the original tube is

$$
T_{\text {rep }}=\frac{L^{2}}{D_{\mathrm{t}}} \cong \frac{\eta_{0} L^{3}}{k T} .
$$

Inserting this result into (3.2) we arrive at :

$$
D_{\text {rot }} \cong \frac{N_{\mathrm{e}} a^{2} k T}{\eta_{0} L^{5}} .
$$

This result may also be derived by a different argument, explained in figure 1 . Here we consider a shorter time interval $\Delta t$ during which the rod moves (on the average) only by one monomer unit $a$, in the direction of its own axis. Thus

$$
\Delta t=a^{2} / D_{\mathrm{t}} \cong \frac{\eta_{0} L a^{2}}{k T} .
$$

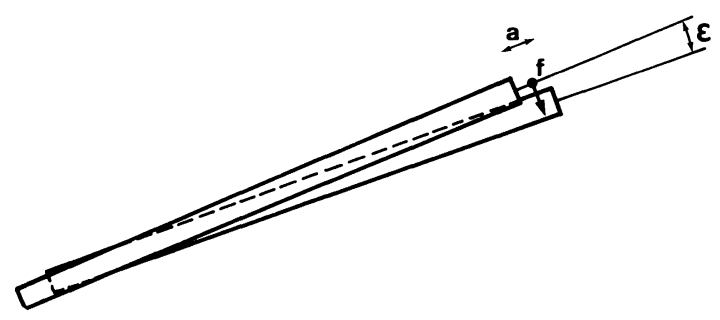

Fig. 1. - Reptation-rotation for a rod : the rod moves by a distance $a$ along its own axis. It then feels the effect of one extra ambient chain, described by a force $f$, or by the resulting torque $\Gamma$. This torque causes a slight angular deflection $\varepsilon$. This process is repeated whenever the rod slides back and forth.

In the portion of tube created (of length $a$ ) the rod is subjected to some random forces $f$, due to the ambient chains, with a component $f_{\perp}$ normal to the rod axis. The corresponding torque is $\Gamma=\frac{L}{2} f_{\perp}$. It is relatively easy to find out what is the rotation angle $\varepsilon$ induced by the torque $\Gamma$, for a needle embedded in an elastic medium of modulus $E_{0}$. Again omitting logarithmic factors, we find :

$$
\varepsilon \cong \frac{\Gamma}{E_{0} L^{3}} \cong \frac{f_{\perp}}{E_{0} L^{2}}
$$


The average of $f_{\perp}$ vanishes, but the average square does not. Simple Gaussian estimates suggest

$$
\left\langle f_{\perp}^{2}\right\rangle=\left(\frac{k T}{R_{\mathrm{e}}}\right)^{2} .
$$

We can now write

$$
D_{\text {rot }}=\frac{\left\langle\varepsilon^{2}\right\rangle}{\Delta t} .
$$

Then inserting equation $(3.7,8)$ into $(2.9)$ we recover equation (3.5).

3.2 COMPARISON WITH STOKES FRICTION. - Equation (3.5) yields a rotational time $\left(\tau_{\text {rot }} \cong D_{\text {rot }}^{-1}\right)$ which becomes astronomically large for long rods. However, in this limit another process may become important. Indeed we may expect that continuum arguments become valid for large $L:$ we then ignore the detailed structure of the molten chains, and describe them as a highly viscous fluid of viscosity $\eta(P)$. The corresponding rotational time is the «Stokes time » [6] :

$$
\tau_{\mathrm{s}} \cong \frac{\eta(P) L^{3}}{k T} \cong \tau_{0} P^{3} N^{3} / N_{\mathrm{e}}^{2}
$$

Again in equation (3.10) we omit logarithmic factors. Comparing (3.10) with equation (3.5) we see that the Stokes process is faster than rotation-reptation whenever $N$ is above a certain threshold $N^{*}$

$$
N^{*} \cong P^{3 / 2} N_{\mathrm{e}}^{-1 / 2} \text {. }
$$

On the other hand, for $N<N^{*}$, the Stokes process is not important.

3.3 TUBE RENEWAL. - In this process, certain ambient chains switch from a position just " below " the solute molecule to a position just «above » thereby modifying the tube. This has discussed in detail by various authors $[8,3]$, and the argument is not seriously modified when we go from a flexible solute to a rigid solute. If we describe tube renewal in terms of a local jump frequency $\tilde{W}$ for the tube, we find that this $\tilde{W}$ is small and very similar to the inverse terminal time $\tilde{W} \sim \tau^{-1}(P)$. This can also be translated in terms of a local friction coefficient $\zeta$ for transverse motions of the tube. $\zeta$ is proportional to $\tau(P)$, and thus to the bulk viscosity $\eta(P)$. This in turn leads to a rotational time which is not qualitatively different from the Stokes time. Thus we may probably omit the process of tube renewal.

4. Probes for the rotational motions. - 4.1 INDIRECT EFFECT OF ROTATIONS ON DRIFT EXPERIMENTS. Our discussion of the translational mobility in section 2 was restricted to a simple steady state situation, where a force $\mathbf{f}$ is applied to a uniform, dilute suspension of rods. With slightly more general conditions, we could in principle measure rotation times.
Assume for instance that originally the rods are oriented at random, and located in a thin region around $x=0$ ( $x$ being an axis parallel to the driving force $\mathrm{f}$ ). At a later time $t$, because of the distribution in orientational angles $\theta$, the rods will have a certain spatial distribution $p_{t}(x)$, which may be probed by various sensitive techniques (e.g. : fluorescence). We expect two limiting regimes for $p_{\mathrm{t}}(x)$ :

a) If $t<\tau_{\text {rot }}$ each rod retains a fixed value of $\cos \theta=u$ and has a velocity along $x$ equal to $\mu_{\|} f u^{2}$, as discussed above. Then

$$
p_{t}(x)=\left\langle\delta\left(x-\mu_{\|} f u^{2}\right)\right\rangle=\int_{0}^{1} \mathrm{~d} u \delta\left(x-\mu_{\|} f u^{2}\right)
$$

giving :

$$
p_{\mathrm{t}}(x)= \begin{cases}1 / 2\left(x \mu_{\|} f\right)^{-1 / 2} & \left(x<\mu_{\|} f\right) \\ 0 & \left(x>\mu_{\|} f\right) .\end{cases}
$$

$b)$ If $t \gg \tau_{\text {rot }}$ the length $x$ travelled by a given rod is roughly the superposition of $t / \tau_{\text {rot }}$ independent increments, and $p_{t}(x)$ is thus Gaussian.

$$
p_{\mathrm{t}}(x)=\text { const. } \exp \left[-\left(x-\bar{x}_{\mathrm{t}}\right)^{2} / 2 \lambda^{2}\right]
$$

where $\bar{x}_{\mathrm{t}}$ corresponds to the average drift mobility

$$
\bar{x}_{\mathrm{t}}=\frac{1}{3} \mu_{\|} f . t
$$

and where the width $\lambda$ of the distribution is given by

$$
\lambda^{2}=\left\langle x^{2}\right\rangle-\bar{x}_{t}^{2} .
$$

Writing

$$
\left\langle x^{2}\right\rangle=\iint_{0}^{t} \mathrm{~d} t_{1} \mathrm{~d} t_{2}\left(\mu_{\|} f\right)^{2}\left\langle u^{2}\left(t_{1}\right) u^{2}\left(t_{2}\right)\right\rangle
$$

and separating the average parts $\left\langle u^{2}\right\rangle=1 / 3$ we get

$$
\lambda^{2}=\left(\mu_{\|} f\right)^{2} \iint_{0}^{t} \mathrm{~d} t_{1} \mathrm{~d} t_{2}\left\langle Q\left(t_{1}\right) Q\left(t_{2}\right)\right\rangle
$$

where $Q=u^{2}-1 / 3$. For Brownian rotations the correlation function for this quadrupolar operator is a simple exponential [9]

$$
\left\langle Q\left(t_{1}\right) Q\left(t_{2}\right)\right\rangle=\left\langle Q^{2}\right\rangle \exp \left(-6 D_{\text {rot }} t\right)
$$

where the factor $6=l(l+1)$ comes from the fact that $Q$ is a spherical harmonic of rank $l=2$. Then, restricting our attention to the regime $6 D_{\text {rot }} t \gg 1$ we may simplify (4.5) and get

$$
\begin{aligned}
\lambda^{2} & =\left(\mu_{\|} f\right)^{2} 2\left\langle Q^{2}\right\rangle t /\left(6 D_{\text {rot }}\right) \\
\left\langle Q^{2}\right\rangle & =\int_{0}^{1} \mathrm{~d} u\left(u^{2}-1 / 3\right)^{2}=\frac{4}{45} .
\end{aligned}
$$


Thus we arrive at

$$
\lambda^{2}=\frac{4}{135}\left(\mu_{\|} f\right)^{2} \frac{t}{D_{\text {rot }}} .
$$

Thus, in this regime, a measurement of the width of the concentration profile can give $D_{\text {rot }}$. The orders of magnitude are not completely unreasonable : the essential parameter is the length $x_{\text {rot }}$ associated with drift during a time $\tau_{\text {rot }}: x_{\text {rot }}=\mu_{\|} f \tau_{\text {rot }}$. Using for instance a charged rod, with one unit of charge $q$ per monomer under an electric field $\mathbf{E}$, and choosing the rotation-reptation regime (Eq. 3.5) we arrive at :

$$
x_{\text {rot }} \cong \frac{q E a}{k T} \frac{a N^{5}}{6 N_{\mathrm{e}}}
$$

choosing $E=1000 \mathrm{~V} / \mathrm{cm}$ and $a=3 \AA$ gives $\frac{q E a}{k T} \sim 10^{-3}$ at room temperature. With $N=N_{\mathrm{e}}=300$ we then get $x_{\text {rot }} \sim 1 / 2 \mathrm{~mm}$. Of course the main worry in this approach would result from spatial inhomogeneities in the matrix, which would add a stray component to the width $\lambda$.

4.2. EleCtric FIELDS. - Applying a field E, we can measure the rotational diffusion constant $D_{\text {rot }}$, using various schemes of detection : either the pure dielectric response, or - more efficiently the electric birefringence (Kerr effect). Experiments of this second type has been performed recently on polybenzyl glutamate (and isocyanate) rods in polystyrene solutions [10]. The data deal mainly with the dependence of $\tau_{\text {rot }}$ on the concentration of $P$ chains in solution, and are thus not directly relevant to our analysis. However, at sufficiently high concentrations, where entanglements should be dominant, $\tau_{\text {rot }}$ becomes independent of $\eta(P)$. This may correspond to our regime $N<N^{*}$. On the other hand, the dependence of $\tau_{\text {rot }}$ on the rod length has not yet been measured in detail.

4.3 VisCoelasticity. - Let us consider again a dilute system of rods (without any significant aggregation). Each rod is assumed to be undeformable. The solution may be characterized either by the volume fraction $\varphi$ of the rods $(\varphi \ll 1)$ or by the number of rods per $\mathrm{cm}^{3} c_{\text {rod }} \sim \frac{\varphi}{N a^{3}}$. Another interesting parameter is obtained by assigning a sphere of influence to each rod : this is a sphere of diameter $L$ with its centre at the mid point of the rod. The fraction of the volume $m$ occupied by these spheres of incluence is

$$
m \cong c_{\text {rod }} L^{3} \sim \varphi N^{2}
$$

consider for instance a high frequency shear experiment, where the melt behaves as an elastic medium of modulus $E_{0}$. Then the macroscopic modulus of the suspension $E$ is increased by the rods, and

$$
\frac{E-E_{0}}{E_{0}} \sim m
$$

i.e., each rod, although it is very thin, succeeds in suppressing most of the strains inside its own sphere of influence. (Eq. (4.2) also omits certain logarithmic factors.)

Having defined these basic parameters, let us now consider a stress relaxation experiment. Here we assume that a small strain $e$ is applied at time $t=0$ to a macroscopic sample, and is kept constant afterwards. We look at the macroscopic stress response $\sigma(t)$. This may be divided into a contribution from the pure melt $\sigma_{0}(t)$ and a correction due to the rods $\delta \sigma(t)$. We are interested in the linear response function

$$
K(t)=\frac{1}{e} \delta \sigma(t)
$$

The viscosity increment is simply

$$
\delta \eta=\int_{0}^{\infty} K(t) \mathrm{d} t .
$$

The qualitative aspect of $K(t)$ is shown on figure 2, where we have assumed that the dominant rotational

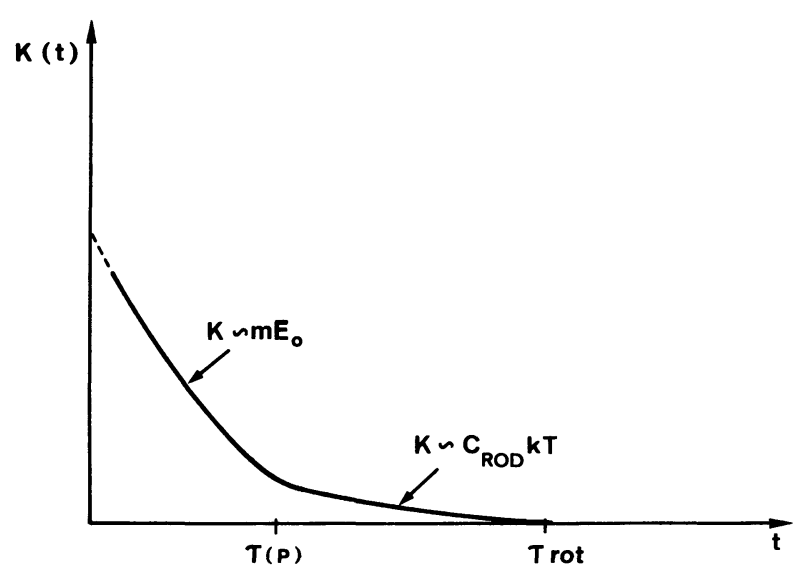

Fig. 2. - Stress response for a unit jump in strain. The response $K(t)$ is the difference between the response of a melt doped by stiff rods and of the pure melt. At times $t<\tau(P)$ the system behaves like an elastic composite. At times $t>\tau(P)$ it behaves like a suspension of rods in a liquid. The area below the curve gives the static viscosity increment $\delta \eta$.

time $\left(\tau_{\text {rot }}=1 / 6 D_{\text {rot }}\right)$ is longer than $\tau(P)-$ this being indeed the most usual situation. The response function contains two parts :

a) up to time $t \sim \tau(P)$ the chains behave like a rubber and we expect from equation (4.2) that

$$
K(t) \sim m E_{0} \quad(t \approx \tau(P)) ;
$$

b) at later times the chains behave like a liquid, and the elastic response drops out. There remains, however, a small but persistent response due to rod alignment in the strain $e$

$$
K(t) \cong c_{\text {rod }} k T \exp -\left(6 D_{\text {rot }} t\right) \quad(t>\tau(P)) .
$$


Equation (4.5) is well known; it may be rederived, if desired, from a Kubo formula expressing $K(t)$ as a stress-stress correlation function. The stress tensor associated with one rod is proportional to $\left(u_{\alpha} u_{\beta}-\frac{1}{3} \delta_{\alpha \beta}\right)$ [11] where $\mathbf{u}$ is a unit vector along the rod axis. The factor $6=l(l+1)$ again corresponds to the relaxation of this second rank tensor $(l=2)$, as in equation (4.6).

The two contributions (Eqs. $(4.14,15))$ may be superposed in the (approximate but convenient) form

$K(t)=m E_{0} \exp [-t / \tau(P)]+c_{\text {rod }} k T \exp \left[-6 D_{\text {rot }} t\right]$.

Note that from equations (4.1) and (1.1) we have :

$$
\frac{c_{\text {rod }} k T}{m E_{0}} \sim \frac{N_{\mathrm{e}}}{N^{3}} \ll 1 \text {. }
$$

Thus the presence of the second term is not an important correction to equation (4.4) when $t<\tau(P)$.

From equation (4.6) the viscosity increment at all frequencies $\omega$ is easily derived

$\delta \eta(\omega)=\frac{m E_{0} \tau(P)}{1+i \omega \tau(P)}+\frac{c_{\text {rod }} k T \tau_{\text {rot }}}{1+i \omega \tau_{\text {rot }}} \quad\left(\tau_{\text {rot }}>\tau(P)\right)$.

Of course in actual fact : (i) there are numerical prefactors in front of both terms, (ii) the relaxation spec- trum of the $P$ chains is not described by a single $\tau(P)$. But the essential physical features are probably present. Because two processes compete for $\tau_{\text {rot }}$, we must distinguish two regimes :

a) If $N>N^{*}$ (long rods) Stokes relaxation dominates : $\tau_{\text {rot }}$ is given by equation (3.10). It is interesting to note that in this case the two contributions to the static viscosity $\delta \eta$ are comparable

$$
\delta \eta \cong m E_{0} \tau(P) \cong c_{\text {rod }} k T \tau_{\mathrm{s}}=m \eta(P) .
$$

Thus $\delta \eta / \eta \sim m$ in agreement with the Stokes behaviour for long rods. Notice that the Stokes prediction works for $\delta \eta$, while it does not work for the translational mobility. Similar effects have been found for flexible solutes in a certain interval of molecular weights [3].

b) If $N<N^{*}$, $\tau_{\text {rot }}$ is given by equation (3.5) and is smaller than $\tau_{\mathrm{s}}$. Then, in the static limit, the second term of equation (4.17) becomes negligible, but the first term is still enough to give us $\frac{\delta \eta}{\eta} \sim m$. Thus we do not expect a very dramatic change in $\delta \eta(\omega=0)$ at the crossover point $\left(N=N^{*}\right)$. But viscoelastic measurements at finite $\omega$ would of course provide an excellent check on our general picture.

Acknowledgments. - The author has greatly benefited from discussions with $\mathrm{H}$. Frisch on translational properties and with E. Marchal and G. Weill on rotational properties.

\section{References}

[1] Gupta, A., Benort, H., Marchal, E., Eur. Polym. J 15 (1979) 285.

[2] Marchal, E., Digest of litterature on dielectrics 40, chap. 13 (1976) ; 41, chap. 14 (1977).

[3] Daoud, M., De Gennes, P. G., J. Polym. Sci. (Physics) 17 (1979) 1971.

[4] Graessley, W., Adv. Polym. Sci. 16 (1974) 1. Graessley, W., J. Polym. Sci. (Physics) 18 (1980) 27.

[5] De Gennes, P. G., J. Chem. Phys. 55 (1971) 572.

De GeNnes, P. G., Scaling concepts in polymer physics (Cornell University Press, Ithaca, NY) 1979.
[6] See for instance Landau-Lifshits, Fluid Mechanics (Pergamon) 1959, p. 68.

[7] De Gennes, P. G., C. R. Hebd. Séan. Acad. Sci. (Paris) 288 (1979) 219.

[8] KLEIN, J., Macromolecules 11 (1978) 852.

[9] See Abragam, A., Principles of nuclear magnetism (Oxford) 1961, p. 299.

[10] Coles, H. J., Weill, G., Eur. Polym. J., to be published.

[11] DoI, M., Edwards, S. F., J. Chem. Soc., Faraday II 74 (1978) $568,918$. 